\title{
Inflammatory myofibroblastic tumor of the trachea: a case report and review of the literature
}

\begin{abstract}
Inflammatory myofibroblastic tumor is a rare tumor with intermediate potential of malignancy usually encountered in children and young adults and located in respiratory system, abdominopelvic and retroperitoneal areas. The most commonly seen in the lungs in respiratory system, trachea involvement is rare. Etiology and pathogenesis of IMT is still uncertain. IMT can mimic various benign or malignant tumors due to absence of specific clinical or radiologic findings. In this study, we presented a case of tracheal IMT detected in a 6 years old girl and discussed its clinical and pathological characteristics.
\end{abstract}

Keywords: Inflammatory myo fibroblastic tumor, trachea, rare
Volume 4 Issue 5 - 2017

\section{Findik S,' Altınok $T^{2}$}

'Department of Pathology, Necmettin Erbakan University, Turkey ${ }^{2}$ Department of Thoracic Surgery, Necmettin Erbakan University, Turkey

Correspondence: Siddika Findik, Department of Pathology, Meram Faculty of Medicine, Necmettin Erbakan University, 42080 Akyokus, Konya, Turkey, Tel +90 332 2236289, Fax +90 332223 6182,Email drpatolog78@gmail.com

Received: February 21, 2017| Published: November 17, 2017

\section{Introduction}

Inflammatory myofibroblastic tumor (IMT) is a rare tumor with intermediary potential of malignancy formerly called inflammatory pseudotumor, plasma cell granuloma, xanthogranuloma or fibrous histiocytoma. ${ }^{1,2}$ Although IMTs are most frequently seen in the respiratory system of the lungs, they are rarely seen in trachea. ${ }^{3}$ These tumors are often seen in the first decade of life and youth and they may be confused with other benign and malignant tumors. ${ }^{4,5}$ Therefore, it should be kept in mind in the differential diagnosis of children with a mass in their respiratory system. Our patient, who has admitted to our clinic with wheezing and difficulty in breathing and diagnosed with IMT as a result of detecting trachea mass lesion in the radiological examination, was presented the case with clinical and pathologic characteristics.

\section{Case report}

A six year old girl admitted to Necmettin Erbakan University Pediatric Emergency Department with difficulty in breathing started recently and wheezing while breathing started 5-6 weeks ago. The patient was given antibiotherapy. Due to the continuation of the complaints, she was transferred to the pediatry department and hospitalized for further treatment and diagnosis. In the thorax CT scans, a polypoid mass, which is $13 \mathrm{~mm}$ in diameter and located at $2 \mathrm{~cm}$ distal of vocal cords, significantly narrowing the lumen of the trachea was detected. She was transferred to the thoracic surgery clinic for surgical treatment. The removed mass was sent to the Pathology Department with a preliminary diagnosis of mucoepidermoid carcinoma. In the macroscopic examination of the mass, which was $1.5 \times 1.5 \times 0.5 \mathrm{~cm}$ in size with roundish and relatively smooth surface, its cross section was observed as reddish and almost homogenous. In the microscopic examination of the tissues, encapsulated tumoral development that created polypoid mass in the bronchial lumen was observed. The tumor was formed by fascicles structures of focal atypic cells in large areas with oval-storiform nuclei and pink fibrillary cytoplasm in vascularized stroma and their storiform pattern. The presence of inflammatory cells including lymphocyte and plasmocyte as well as neutrophils, eosinophils and multinucleated giant cells in less amounts was noticed.

There was no atypical mitosis in 10 high power field. In immunohistochemical examination; vimentin and smooth muscle actin (SMA) diffuse positive, desmine focal weakly positive, S-100, CD-31, CD 99, CD-57, P-63, anaplastic lymphoma receptor tyrosine kinase gene (ALK) and myoglobin was negative. Ki-67 proliferation index was below 5\% (Figure 1). In line with these findings, the patient was diagnosed with "Inflammatory Myofibroblastic Tumor". No adjuvant therapy was needed since the patient was under clinical follow-up after total excision.

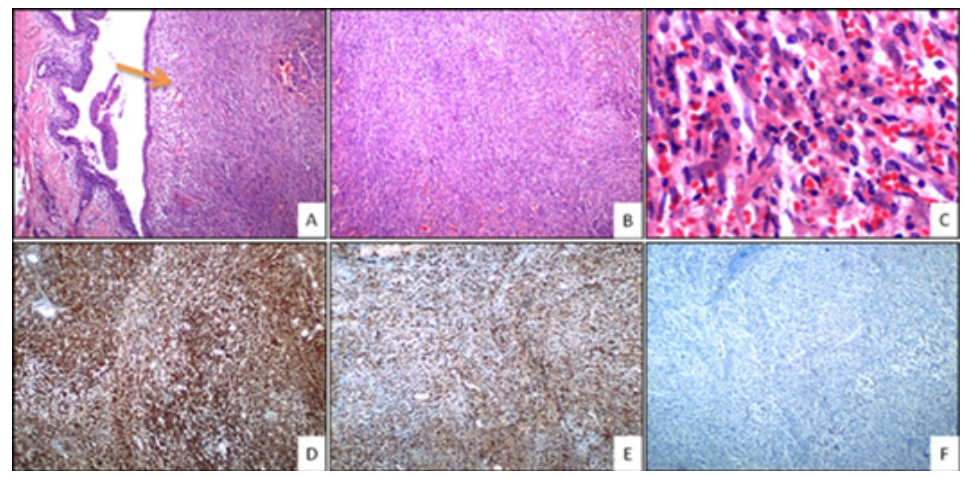

Figure I A: Polypoid tumoral mass under bronchial epithelium (Orange arrow) (H\&E 40x magnification); B: Tumor cells with fascicle and storiform growth patterns (H\&E 40x magnification); C: Inflammatory cells (eosinophils, neutrophils, plasmocytes and lymphocytes) in the tumor (H\&E 400x magnification); D: Positivity of immunohistochemical vimentin in tumor cells (H\&E 40x magnification); E: Positivity of immunohistochemical SMA in tumor cells (H\&E 40x magnification); F: Negativity of immunohistochemical ALK in tumor cells (H\&E 40x magnification). H\&E, Hematoxylin and eosin staining. 


\section{Discussion}

IMT is a rare mesenchymal tumor that is still not fully understood in terms of its etiology and pathogenesis. ${ }^{6,7}$ According to several studies conducted in recent years, infection, surgery, trauma and chromosomal variations may have a role in the development of IMT and there are localized deviations in chromosome 2 and 9.6,8

IMT was first described in 1939 and accepted as a benign lesion; however, according to the case series reported within the last 20 years, the disease has a potential of aggressive trending as local recurrence rate was $37 \%$ and metastases rate was $11 \% .{ }^{5}$ According to the classification of the World Health Organization (2002), IMT was classified as a tumor with potential of intermediary malignancies and rare metastasis. ${ }^{5,9}$ It was called inflammatory pseudotumor in the past years since it might behave as act malignant clinically, radiologically and microscopically. ${ }^{10}$ The average age of patients with IMT is 9 and it is often seen in children and young adults and it is slightly more common in girls than in boys. ${ }^{5,8}$ Our patient was a 6-year-old girl and it was consistent with the literature.

Although IMT can occur in many different anatomical regions, it is most frequently seen in the lungs of the respiratory system followed by abdominopelvic region and retroperitoneum. .,6,10 $^{5}$ Tracheal involvement is rare. The primary tumors of trachea are very rare. Tumors seen in adults are usually malignant, whereas in childhood usually benign. The most common types of malignant tumors are squamous cell carcinoma, adenoid cystic carcinoma and mucoepidermoid carcinoma; while polyps, chondroid hamartoma and IMTs are common types of benign tumors. The tracheal involvement rate of IMT ranges from $0.04 \%$ to $0.07 \%$ among all respiratory tract tumors. ${ }^{3}$ Gaissert et al. ${ }^{11}$ have reported only 1 IMT case among 90 cases of primary tracheal tumors in the last 44 years. ${ }^{11}$ Between the years of 1974-2007, in 26 IMT cases of Fabre et al; only one of them was diagnosed with tracheal IMT. ${ }^{12}$

Although the diameter size of IMTs ranges from $2 \mathrm{~cm}$ to $20 \mathrm{~cm}$, the average diameter size is between 5 and $10 \mathrm{~cm} .{ }^{1,2}$ They are in the form of lobular, multinodular and hard masses macroscopically. Its cross sections may be gray-tan or reddish and their macroscopic views may be like many other benign and malignant neoplasms such as leiomyoma, leiomyosarcoma, inflammatory liposarcoma. ${ }^{5,9}$ In our case, the mass was $1.5 \mathrm{~cm}$ in diameter with a polypoid appearance radiologically, and it had a smooth and soft surface.

Since clinical and radiological findings are nonspecific in the diagnosis of IMTs, the definitive diagnosis is based on histopathological examination of the excised material. ${ }^{6,13}$ Histologically; infiltration of inflammatory cell consisting of plasma cells, lymphocytes and eosinophils that are accompanying the proliferation of myofibroblasts is observed. ${ }^{1,5}$ Neoplastic myofibroblasts form either fascicular or storiform patterns. ${ }^{1,2}$ Necrosis and calcification may rarely occur. ${ }^{1}$ Focal cellular atypia and mitosis can be seen, but no atypical mitosis is expected..$^{2,10}$ In our case, a cellular tumor forming fascicular and storiform patterns was observed. No necrosis and calcification was seen. The mild cellular atypia was present, but no atypical mitosis was detected.

Immunohistochemical studies are important to the differential diagnosis of IMT. There is a positive expression with vimentin, actin and rarely desmine in the tumor cells. ${ }^{2,5,10,14}$ In our case, vimentin and SMA was diffuse positive and desmine was focal weakly positive.
It was differentiated from other malignant mesenchymal tumors with low Ki-67 proliferation index, lack of atypical mitosis and pleomorphism. The presence of inflammatory cells were also helpful in differential diagnosis. In the pathogenesis of disease, the role of mutation of anaplastic lymphoma kinase (ALK) gene was shown and there is a cytoplasmic positivity with ALK immunohistochemically in about $50 \%$ of the cases. ${ }^{5,7,14,15}$ In our case, no ALK positivity was found. In several studies, ALK negative IMTs showing more aggressive clinical behaviors due to the increased risk of metastasis and local recurrence are reported. ${ }^{1,5,10,13,14}$ This suggests that detection of ALK with immunohistochemical method may be used to determine the prognosis.

Total surgical excision is curative for the treatment of IMT. Therefore, it is very important to differentiate from sarcoma and make correct diagnosis in order to avoid unnecessary aggressive treatment. ${ }^{13}$ Fabre et al reported a 10-year survey with a rate of $89 \%$ in IMTs after total resection. Adjuvant treatment after surgery has been reported to be useful in recurrence or incomplete resection. ${ }^{12}$ Although the relationship between ALK expression and clinical behavior of the tumor is not clear, detection of ALK may be important in order to apply adjuvant treatment to the patients. ${ }^{5}$ In addition, there are some studies suggesting that more aggressive treatment regimen may be needed in ALK negative patients. ${ }^{14}$ Curative surgical treatment was applied to our case and adjuvant therapy didn't considered because of no residue tumor within surgical margins of the microscopic examination. However the patient was under close clinical follow-up.

\section{Conclusion}

IMT is an entity that should be kept in mind in the differential diagnosis of the masses in the respiratory system especially during childhood. Since it may be confused with many different benign and malignant tumors clinically and radiologically, the definitive diagnosis is possible with histopathologic examination after excision. Making differential diagnosis with other malignancies will be useful in terms of preventing unnecessary aggressive treatment. In ALKnegative patients, different treatment approaches will be needed in order to longer clinical follow-up studies.

\section{Acknowledgments}

The present work was performed after taking informed consent from the patient and a sincere effort has been made to uphold patient confidentiality.

\section{Conflicts of interest}

The authors have declared that no competing interests exist. Financial assistance/funding Nil.

\section{References}

1. Fletcher CDM. Tumors of Soft Tissue Chapter 24 in Diagnostic Histopathology of Tumors. ( $4^{\text {th }}$ edn), Elsevier Saunders USA. 2013;18231824.

2. Weiss SW, Goldblum JR. Fibrous tumors of infancy and childhood. In: Weiss SW \& Goldblum JR, editors. Enzinger and Weiss's Soft Tissue Tumors. $5^{\text {th }}$ edn, Mosby Elsevier, Philadelphia, USA, 2008;284-289.

3. De Palma A, Loizzi D, Sollitto F, et al. Surgical treatment of a rare case of tracheal inflammatory pseudotumor in pediatric age. Interact Cardiovasc Thorac Surg 2009;9:1035-1037. 
4. Tomazic A, Gvardijancic D, Maucec J, et al. Inflammatory myofibroblastic tumor of the Pancreatic head-a case report of a 6 months old child and review of the literature. Radiol Oncol 2015;49(3):265-270.

5. Kim S, Gamez J, Okuno S, et al. Abdominopelvic inflammatory myofibroblastic tumor that metastasized to the vertebrae and liver: A case report and review of the literature. 2015;12:9-12.

6. Xu LF, Zhou J, Liang CZ. Inflammatory Myofibroblastic Tumor of Genitourinary Tract Beyond Collecting System: A Rare Case Report With Literature Review Medicine. Medicine (Baltimore). 2015;94(42):e1706.

7. Markopoulos C, Charalampoudis P, Karagiannis E, et al. Inflammatory Myofibroblastic Tumor of the Breast. Case Rep Surg: 705127; 2015.

8. Chen W, Jiang Z, Zhou F, et al. A large inflammatory myofibroblastic tumor involving both stomach and spleen: A case report and review of the literature. Oncology Letters. 2015;9(2):811-815.

9. Dervisoglu S. Yumusak Doku Tumorleri Biyopsilerinin Yorumu. Nobel Tip Kitabevleri. 2014;99-102.

10. Humidi A, AL-Khamiss A. Inflammatory Myofibroblastic Tumor Arising in the External Ear: Unexpected Location. (Case Report). Int J HealthSci (Qassim) 2015;9(2):201-205.
11. Gaissert HA, Grillo HC, Shadmehr MB, et al. Uncommon primary tracheal tumors. Ann Thorac Surg. 2006;82:268-272.

12. Fabre D, Fadel E, Singhal S, et al. Complete resection of pulmonary inflammatory pseudotumors has excellent long term prognosis. $J$ Thorac Cardiovasc Surg. 2009;137(2):435-440.

13. Jain A, Kasana S, Ramrakhiani D, et al. Inflammatory myofibroblastic tumor of the stomach in an adult female - report of a rare case and review of the literature. Turk J Gastroenterol. 2012;23(4):399-405.

14. Hansen C, Eisenbach C, Torres C, et al. Maxillary Sinus Inflammatory Myofibroblastic Tumors: A Review and Case Report. Oncol Med. 2015;1-9.

15. Pierry C, Perot G, Karanian-Philippe M, et al. Polypoid laryngeal inflammatory myofibroblastic tumors: misleading lesions: description of six cases showing ALK over expression. Am J Clin Pathol 2015;144(3):511-516. 